\title{
An Optimized Technique for Wigner Kernel Estimation
}

\author{
Venelin Todorov \\ Bulgarian Academy of Sciences \\ Institute of Mathematics and Informatics \\ ul. G. Bonchev 8, 1113 Sofia, Bulgaria \\ Bulgarian Academy of Sciences \\ Institute of Information and Communication Technologies \\ ul. G. Bonchev 25A, 1113 Sofia, Bulgaria \\ Email: vtodorov@math.bas.bg,venelin@parallel.bas.bg

\section{Ivan Dimov \\ Bulgarian Academy of Sciences} \\ Institute of Information and Communication Technologies \\ ul. G. Bonchev 25A, 1113 Sofia, Bulgria \\ Email: ivdimov@bas.bg
}

\author{
Stefka Fidanova \\ Bulgarian Academy of Sciences \\ Institute of Information and Communication Technologies \\ ul. G. Bonchev 25A, 1113 Sofia, Bulgria \\ Email: stefka@parallel.bas.bg
}

\begin{abstract}
We study an optimized Adaptive Monte Carlo algorithm for the Wigner kernel - an important problem in quantum mechanics. We will compare the results with the basic adaptive approach and other stochastic approaches for computing the Wigner kernel represented by difficult multidimensional integrals in dimension $d$ up to 12. The higher cases $d>12$ will be considered for the first time. A comprehensive study and an analysis of the computational complexity of the optimized Adaptive MC algorithm under consideration has also been presented.
\end{abstract}

\section{INTRODUCTION}

D IFFERENT mathematical formulations of quantum mechanics exist, among which the ones suggested by E. Schrodinger, E. Wigner, R. Feynman, L.V. Keldysh, K. Husimi, D. Bohm are more frequently used nowadays [10]. The Wigner formulation of quantum mechanics allows the comprehension and prediction of quantum mechanical phenomena in terms of quasidistribution functions. One of the best known physicist Richard Feynman formulated the problem of finding an effective and fast algorithm with linear or polynomial computational complexity for computing multidimensional integrals that represent Wigner kernel [6]. More information about the signed particle formulation of a singlebody and many-body system can be found in [8], [9], [10].

Venelin Todorov is supported by the Bulgarian National Science Fund under Project KP-06-M32/2 - 17.12.2019 "Advanced Stochastic and Deterministic Approaches for Large-Scale Problems of Computational Mathematics" and by the National Scientific Program "Information and Communication Technologies for a Single Digital Market in Science, Education and Security (ICT in SES)", contract No DO1-205/23.11.2018, financed by the Ministry of Education and Science in Bulgaria. The work is also supported by Bulgarian National Science Fund under Project DN 12/5-2017 "Efficient Stochastic Methods and Algorithms for Large-Scale Problems" and by the Project KP-06-Russia/17 "New Highly Efficient Stochastic Simulation Methods and Applications" funded by the National Science Fund - Bulgaria.
Up to now the Wigner kernel is calculated with deterministic methods [11], [12], [14] which suffer from the ,curse of dimensionality" [2]. In our previous work [13] we consider the case when the dimension $d \leq 9$, the higher cases $d>12$ will be considered for the first time in the presented work.

\section{Description OF THE OPTIMIZED AdAPTIVE APPROACH}

Adaptive strategy [1], [3], [4] is well known method for evaluation of multidimensional integrals, especially when the integrand function has peculiarities and peaks. Let $p_{j}$ and $I_{\Omega_{j}}$ are the following expressions: $p_{j}=\int_{\Omega_{j}} p(\mathbf{x}) \mathrm{d} \mathbf{x}$ and $I_{\Omega_{j}}=$ $\int_{\Omega_{j}} f(\mathbf{x}) p(\mathbf{x}) \mathrm{d} \mathbf{x}$. Consider now a random point $\xi^{(j)} \in$ $\Omega_{j}$ with a density function $p(\mathbf{x}) / p_{j}$. In this case $I_{\Omega_{j}}=$ $\mathbf{E}\left[\frac{p_{j}}{N} \sum_{i=1}^{N} f\left(\xi_{i}^{(j)}\right)\right]=\mathbf{E} \theta_{N}$. This adaptive algorithm gives an approximation with an error $\varepsilon \leq c N^{-1 / 2}$, where $c \leq$ $0.6745 \sigma(\theta)(\sigma(\theta)$ is the standard deviation).

The optimized adaptive algorithm has higher accuracy than the original Adaptive Monte Carlo algorithm as can be seen from the tables below. The increase of the constant for the initial number of taken subregions $M=8$ improves the relative error compared with the previous choice $M=2$ in [13]. The optimized adaptive algorithm is described below.

\section{Algorithm}

1. Input data: total number of points $N 1$, constant $M=8$ (the initial number of subregions taken), constant $\varepsilon$ (max value of the variance in each subregion), constant $\delta$ (maximal admissible number of subregions), d-dimensionality of the initial region/domain, $f$ - the function of interest. 
1.1. Calculate the number of points to be taken in each subregion $N=N 1 / \delta$.

2. $\quad$ For $j=1, M^{d}$ :

2.1. Calculate the approximation of $I_{\Omega_{j}}$ and the variance $\mathbf{D}_{\Omega_{j}}$ in subdomain $\Omega_{j}$ based on $N$ independent realizations of random variable $\theta_{N}$

2.2. If $\left(\mathbf{D}_{\Omega_{j}} \geq \varepsilon\right)$ then

2.2.1. Choose the axis direction on which the partition will perform,

2.2.2. Divide the current domain into two $\left(G_{j_{1}}, G_{j_{2}}\right)$ along the chosen direction,

2.2.3. If the length of obtained subinterval is less than $\delta$ then go to step 2.2.1 else $j=j_{1} G_{j_{1}}$ is the current domain right and go to step 2.1;

2.3. Else if $\left(\mathbf{D}_{\Omega_{j}}<\varepsilon\right)$ but an approximation of $I_{G_{j_{2}}}$ has not been calculated yet, then $j=j_{2} G_{j_{2}}$ is the current domain along the corresponding direction right and go to step 2.1 ;

2.4. Else if $\left(\mathbf{D}_{\Omega_{j}}<\varepsilon\right)$ but there are subdomains along the other axis directions, then go to step 2.1;

2.5. Else Accumulation in the approximation $I_{N}$ of $I$.

\section{Computational complexity}

For the simple case when we have the two dimensional case $(N=2)$ and on the first step in the optimized adaptive approach we have $M=8$ subdomains in our optimized Adaptive approach and

$$
\hat{\theta}_{N}=\sum_{j=1}^{M} \frac{1}{N_{j}} \sum_{i=j}^{N_{M}} \theta_{i}
$$

where $\sum_{j=1}^{M} N_{j}=N$, so we have the same number of operations as the Crude Monte Carlo, which computational complexity is linear [2], to evaluate an approximation of $I_{G_{j}}$.

So we choose only $\mathcal{O}(1)$ subdomains where the variance is greater than the parameter $\varepsilon$ and this is independent of $N$. When we divide the domain on every step adaptiveness is not in all subdomains, but only in $\mathcal{O}(1)$ subdomains. At the beginning we have to choose $\frac{N}{k_{0}}$ random points. After that when dividing the domain into $2^{N}$ subdomains, we choose only $\mathcal{O}(1)$ subdomains, this choice is again independent of $N$. In these subdomains we choose $\frac{N}{k_{1}}$ points. On the $j^{t h}$ step of the Adaptive approach we choose $\mathcal{O}(1)$ subdomains with $\frac{N}{k_{j}}$ points. We have that $\sum_{j=0}^{i} \frac{1}{k_{j}}=1$. Therefore for the computational complexity we obtain

$$
\frac{N}{k_{0}}+\mathcal{O}(1) \frac{N}{k_{1}}+\cdots+\mathcal{O}(1) \frac{N}{k_{i}}=
$$

$$
=N \mathcal{O}(1)\left(\sum_{j=0}^{i} \frac{1}{k_{j}}\right)=N \mathcal{O}(1)=\mathcal{O}(N) .
$$

In this way we can conclude that the computational complexity of the optimized Adaptive algorithm is linear.

\section{NUMERICAL EXAMPLES}

A new formulation of quantum mechanics in terms of signed classical field-less particles is presented in [7], [10]. Just for completeness we give here the three postulates which completely define the new mathematical formulation of quantum mechanics taken from [7].

Postulate I. Physical systems can be described by means of (virtual) Newtonian particles, i.e. provided with a position $\mathbf{x}$ and a momentum $\mathbf{p}$ simultaneously, which carry a sign which can be positive or negative.

Postulate II. A signed particle, evolving in a potential $V=V(x)$, behaves as a field-less classical point-particle which, during the time interval $\mathrm{d} t$, creates a new pair of signed particles with a probability $\gamma(\mathbf{x}(t)) \mathrm{d} t$, where

$\gamma(\mathbf{x})=\int_{-\infty}^{+\infty} \mathrm{D} \mathbf{p}^{\prime} V_{W}^{+}\left(\mathbf{x} ; \mathbf{p}^{\prime}\right) \equiv \lim _{\triangle \mathbf{p}^{\prime} \rightarrow 0^{+}} \sum_{\mathbf{M}=-\infty}^{+\infty} V_{W}^{+}\left(\mathbf{x} ; \mathbf{M} \triangle \mathbf{p}^{\prime}\right)$,

where $\hbar=\frac{h}{2 \pi}$ is the reduced Planck constant $(h)$ or Dirac constant, $\mathbf{M}=\left(M_{1}, M_{2}, \ldots, M_{d}\right)$ is a set of $d$ integers and $V_{W}^{+}(\mathbf{x} ; \mathbf{p})$ is the positive part of the quantity

$V_{W}(\mathbf{x} ; \mathbf{p})=\frac{i}{\pi^{d} \hbar^{d+1}} \int_{-\infty}^{+\infty} \mathrm{d} \mathbf{x}^{\prime} e^{-\frac{2 i}{\hbar} \mathbf{x}^{\prime} \mathbf{p}}\left[V\left(\mathbf{x}+\mathbf{x}^{\prime}\right)-V\left(\mathbf{x}-\mathbf{x}^{\prime}\right)\right]$,

known as the Wigner kernel (in a d-dimensional space) [15]. If, at the moment of creation, the parent particle has sign $s$, position $\mathbf{x}$ and momentum $\mathbf{p}$, the new particles are both located in $\mathbf{x}$, have signs $+s$ and $-s$, and momentum $\mathbf{p}+\mathbf{p}^{\prime}$ and $\mathbf{p}-\mathbf{p}^{\prime}$ respectively, with $\mathbf{p}^{\prime}$ chosen randomly according to the (normalized) probability $\frac{V_{W}^{+}(\mathbf{x} ; \mathbf{p})}{\gamma(\mathbf{x})}$.

Postulate III. Two particles with opposite sign and same phase-space coordinates $(\mathbf{x}, \mathbf{p})$ annihilate.

The infinite domain of integration can be mapped into the $s$ dimensional unit hypercube using the following transformation $\frac{1}{2}+\frac{1}{\pi} \arctan (x)$ which maps $(-\infty, \infty)$ to $(0,1)$. We want to compute (1) in the $3,6,9$ and for the first time in 12 dimensional case,

$$
V w(x, p)=\int e^{\left(\frac{-i 2 \sum_{k=1}^{n} x_{k}^{\prime} p_{k}}{\hbar}\right)} \times
$$

$\left[V\left(x_{1}+x_{1}^{\prime}, \ldots x_{n}+x_{n}^{\prime}\right)-V\left(x_{1}-x_{1}^{\prime}, \ldots x_{n}-x_{n}^{\prime}\right)\right] d x_{1}^{\prime} \ldots d x_{n}^{\prime}$,

where the Wigner potential is $V=V(x)=$ $\left\{x_{1} \ldots x_{n}, \quad x^{\prime}, x, p, x+x^{\prime}, x-x^{\prime} \in[0,1]\right\}$. It is well known that Wigner kernel has real values [15].

First, we will make a comparison with deterministic method of mid rectangulars, and after that with the well known 
Table I

RELATIVE ERROR OF THE OPTIMIZED ADAPTIVE APPROACH, ADAPTIVE APPROACH AND THE DETERMINISTIC MID RECTANGULAR METHOD

\begin{tabular}{|c|c|c|c|c|c|c|c|}
\hline \hline $\mathrm{s}$ & $\mathrm{N}$ & determ. & $\mathrm{t}(\mathrm{s})$ & OptAdapt & $\mathrm{t}(\mathrm{s})$ & Adapt & $\mathrm{t}(\mathrm{s})$ \\
\hline \hline \multirow{4}{*}{3} & $32^{2} \times 50$ & $8.51 \mathrm{e}-03$ & 0.2 & $1.47 \mathrm{e}-03$ & 0.1 & $2.71 \mathrm{e}-03$ & 0.1 \\
\cline { 2 - 8 } 3 & $32^{2} \times 100$ & $8.21 \mathrm{e}-03$ & 0.5 & $8.84 \mathrm{e}-05$ & 0.31 & $3.42 \mathrm{e}-04$ & 0.2 \\
\cline { 2 - 8 } & $64^{2} \times 50$ & $5.76 \mathrm{e}-03$ & 1 & $2.32 \mathrm{e}-05$ & 0.8 & $7.52 \mathrm{e}-05$ & 0.55 \\
\cline { 2 - 8 } & $64^{2} \times 100$ & $4.89 \mathrm{e}-03$ & 1.9 & $4.23 \mathrm{e}-06$ & 1.7 & $1.21 \mathrm{e}-05$ & 1.3 \\
\hline \hline \multirow{4}{*}{6} & $8^{4} \times 50^{2}$ & $1.16 \mathrm{e}-02$ & 41.2 & $8.64 \mathrm{e}-05$ & 30 & $9.09 \mathrm{e}-04$ & 18.1 \\
\cline { 2 - 8 } & $8^{4} \times 100^{2}$ & $9.75 \mathrm{e}-03$ & 160.6 & $2.31 \mathrm{e}-06$ & 98 & $1.52 \mathrm{e}-05$ & 57.9 \\
\cline { 2 - 8 } & $16^{4} \times 50^{2}$ & $7.84 \mathrm{e}-03$ & 635.2 & $1.53 \mathrm{e}-05$ & 487 & $4.37 \mathrm{e}-04$ & 311.5 \\
\cline { 2 - 8 } & $16^{4} \times 100^{2}$ & $2.12 \mathrm{e}-03$ & 2469.1 & $1.08 \mathrm{e}-05$ & 1657 & $3.80 \mathrm{e}-04$ & 987.1 \\
\hline \hline \multirow{4}{*}{9} & $6^{6} \times 16^{3}$ & $1.75 \mathrm{e}-03$ & 835.5 & $2.66 \mathrm{e}-05$ & 504 & $7.62 \mathrm{e}-05$ & 330.5 \\
\cline { 2 - 8 } & $6^{6} \times 32^{3}$ & $1.35 \mathrm{e}-03$ & 5544.1 & $8.66 \mathrm{e}-06$ & 3451 & $2.73 \mathrm{e}-05$ & 2225.1 \\
\cline { 2 - 7 } & $6^{6} \times 40^{3}$ & $1.12 \mathrm{e}-03$ & 10684.4 & $7.83 \mathrm{e}-07$ & 6531 & $8.12 \mathrm{e}-06$ & 4491.5 \\
\hline
\end{tabular}

stochastic approaches of Sobol QMC and Fibonacci based lattice rule FIBO, see [13].
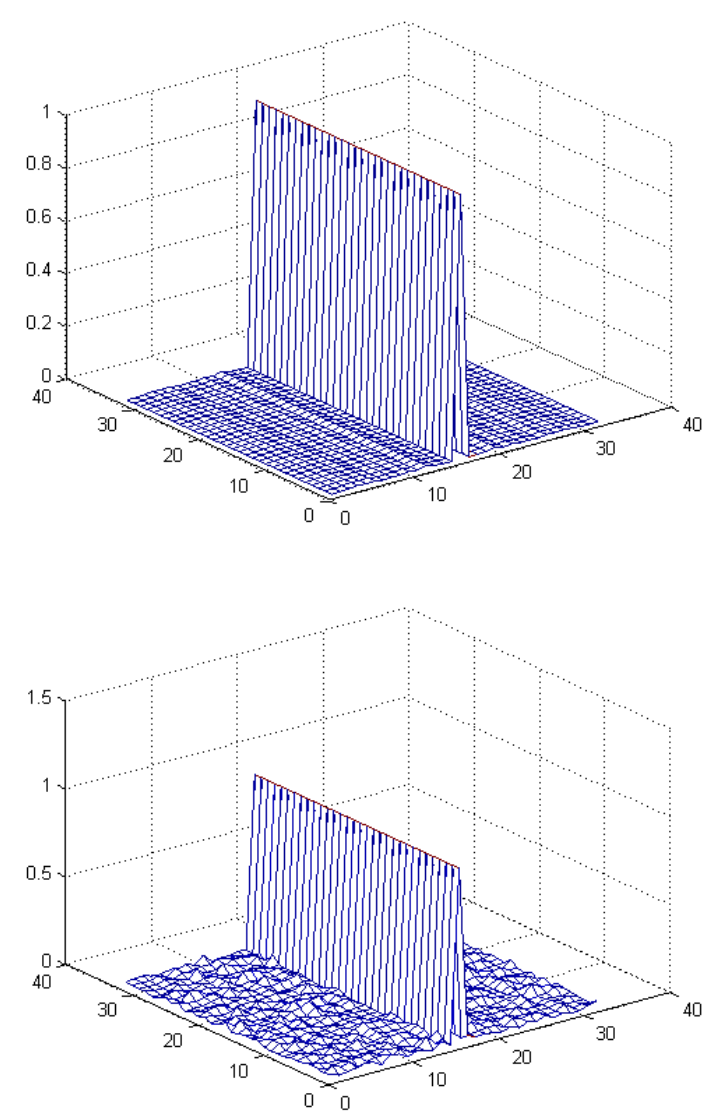

Figure 1. The Wigner kernel with optimized adaptive and standard adaptive method
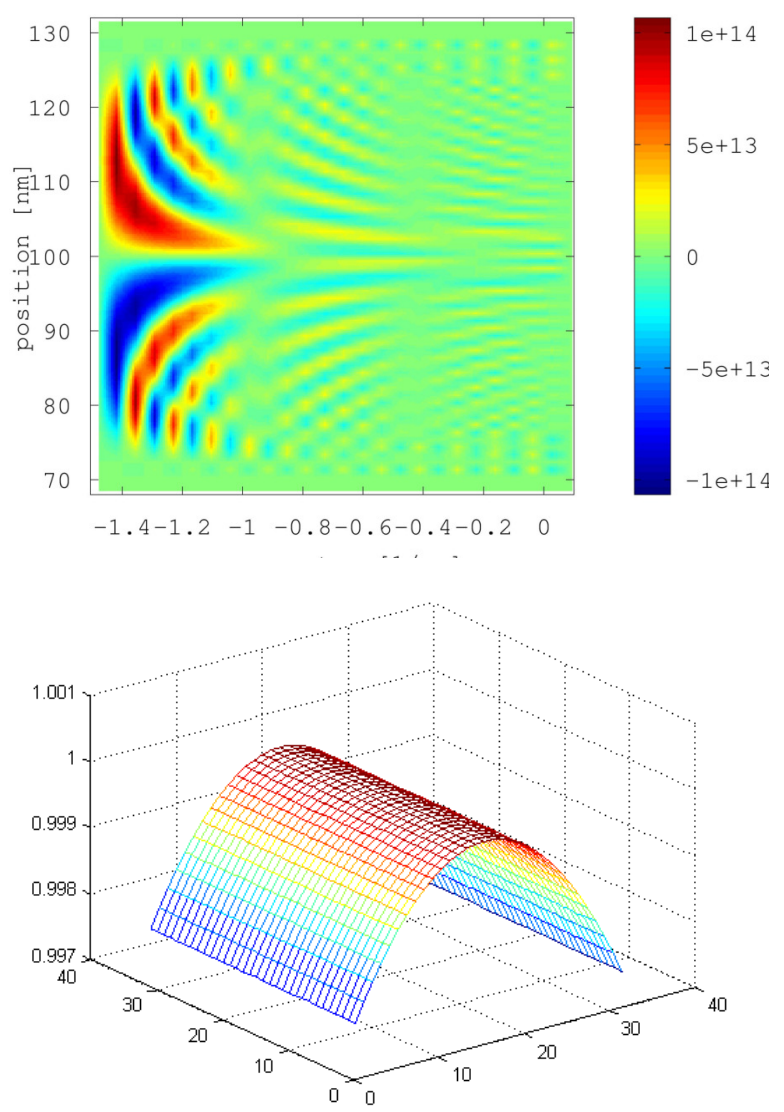

Figure 2. The position and the peak of the Wigner kernel with optimizes adaptive approach

Table II

RELATIVE ERROR FOR 3 DIMENSION

\begin{tabular}{|c|c|c|c|c|}
\hline \hline $\mathrm{N}$ & Adapt & OptAdapt & FIBO & Sobol \\
\hline $10^{3}$ & $5.36 \mathrm{e}-03$ & $3.21 \mathrm{e}-04$ & $3.72 \mathrm{e}-02$ & $1.07 \mathrm{e}-02$ \\
\hline $10^{4}$ & $4.84 \mathrm{e}-04$ & $4.02 \mathrm{e}-05$ & $7.06 \mathrm{e}-03$ & $8.77 \mathrm{e}-03$ \\
\hline $10^{5}$ & $2.51 \mathrm{e}-05$ & $3.33 \mathrm{e}-06$ & $3.40 \mathrm{e}-03$ & $8.57 \mathrm{e}-04$ \\
\hline $10^{6}$ & $1.76 \mathrm{e}-05$ & $1.56 \mathrm{e}-07$ & $1.01 \mathrm{e}-03$ & $6.73 \mathrm{e}-04$ \\
\hline $10^{7}$ & $6.26 \mathrm{e}-06$ & $5.67 \mathrm{e}-08$ & $1.80 \mathrm{e}-04$ & $5.98 \mathrm{e}-05$ \\
\hline
\end{tabular}

Table III

RELATIVE ERROR FOR 6 DIMENSION

\begin{tabular}{|c|c|c|c|c|}
\hline \hline $\mathrm{N}$ & Adapt & OptAdapt & FIBO & Sobol \\
\hline $10^{3}$ & $6.72 \mathrm{e}-03$ & $1.07 \mathrm{e}-04$ & $7.82 \mathrm{e}-03$ & $2.42 \mathrm{e}-02$ \\
\hline $10^{4}$ & $9.10 \mathrm{e}-04$ & $2.11 \mathrm{e}-05$ & $5.01 \mathrm{e}-03$ & $5.02 \mathrm{e}-03$ \\
\hline $10^{5}$ & $5.26 \mathrm{e}-05$ & $3.02 \mathrm{e}-06$ & $6.88 \mathrm{e}-03$ & $4.60 \mathrm{e}-04$ \\
\hline $10^{6}$ & $2.70 \mathrm{e}-06$ & $3.21 \mathrm{e}-07$ & $7.68 \mathrm{e}-04$ & $3.59 \mathrm{e}-04$ \\
\hline $10^{7}$ & $1.03 \mathrm{e}-06$ & $5.13 \mathrm{e}-08$ & $4.12 \mathrm{e}-04$ & $8.11 \mathrm{e}-05$ \\
\hline \hline
\end{tabular}

In Table I it can be seen that the optimized stochastic approach gives better results and lower relative errors than the adaptive approach used in our previous study [13]. It can be seen that the computational time for the optimized Adaptive 
Table IV

RELATIVE ERROR FOR 9 DIMENSION

\begin{tabular}{|c|c|c|c|c|}
\hline \hline $\mathrm{N}$ & Adapt & OptAdapt & FIBO & Sobol \\
\hline $10^{3}$ & $4.92 \mathrm{e}-02$ & $6.11 \mathrm{e}-04$ & $2.03 \mathrm{e}-02$ & $5.42 \mathrm{e}-02$ \\
\hline $10^{4}$ & $9.09 \mathrm{e}-04$ & $1.12 \mathrm{e}-05$ & $2.02 \mathrm{e}-03$ & $6.02 \mathrm{e}-03$ \\
\hline $10^{5}$ & $3.32 \mathrm{e}-05$ & $9.88 \mathrm{e}-07$ & $9.16 \mathrm{e}-04$ & $3.57 \mathrm{e}-03$ \\
\hline $10^{6}$ & $6.46 \mathrm{e}-06$ & $2.01 \mathrm{e}-07$ & $7.13 \mathrm{e}-04$ & $8.02 \mathrm{e}-04$ \\
\hline $10^{7}$ & $1.21 \mathrm{e}-06$ & $5.84 \mathrm{e}-08$ & $4.84 \mathrm{e}-04$ & $5.19 \mathrm{e}-04$ \\
\hline
\end{tabular}

Table V

RELATIVE ERROR FOR 12 DIMENSION

\begin{tabular}{|c|c|c|c|c|}
\hline \hline $\mathrm{N}$ & Adapt & OptAdapt & FIBO & Sobol \\
\hline $10^{3}$ & $3.91 \mathrm{e}-03$ & $1.11 \mathrm{e}-04$ & $1.33 \mathrm{e}-02$ & $2.85 \mathrm{e}-02$ \\
\hline $10^{4}$ & $5.04 \mathrm{e}-04$ & $9.01 \mathrm{e}-06$ & $1.34 \mathrm{e}-03$ & $4.04 \mathrm{e}-03$ \\
\hline $10^{5}$ & $2.76 \mathrm{e}-04$ & $3.14 \mathrm{e}-06$ & $5.51 \mathrm{e}-04$ & $1.77 \mathrm{e}-03$ \\
\hline $10^{6}$ & $4.14 \mathrm{e}-05$ & $1.12 \mathrm{e}-07$ & $4.43 \mathrm{e}-04$ & $4.07 \mathrm{e}-04$ \\
\hline $10^{7}$ & $2.31 \mathrm{e}-06$ & $2.83 \mathrm{e}-08$ & $2.5684 \mathrm{e}-04$ & $2.7 \mathrm{e}-04$ \\
\hline \hline
\end{tabular}

Table VI

RELATIVE ERROR FOR 15 DIMENSION

\begin{tabular}{|c|c|c|c|c|}
\hline \hline t,s & Adapt & OptAdapt & FIBO & Sobol \\
\hline $10^{3}$ & $6.21 \mathrm{e}-03$ & $6.22 \mathrm{e}-04$ & $8.76 \mathrm{e}-04$ & $2.31 \mathrm{e}-02$ \\
\hline $10^{4}$ & $4.45 \mathrm{e}-04$ & $4.51 \mathrm{e}-05$ & $5.56 \mathrm{e}-04$ & $5.45 \mathrm{e}-03$ \\
\hline $10^{5}$ & $5.43 \mathrm{e}-05$ & $3.56 \mathrm{e}-06$ & $3.34 \mathrm{e}-04$ & $4.11 \mathrm{e}-03$ \\
\hline $10^{6}$ & $1.23 \mathrm{e}-05$ & $4.16 \mathrm{e}-07$ & $1.34 \mathrm{e}-04$ & $6.45 \mathrm{e}-04$ \\
\hline
\end{tabular}

Table VII

RELATIVE ERROR FOR 18 DIMENSION

\begin{tabular}{|c|c|c|c|c|}
\hline \hline $\mathrm{t}, \mathrm{s}$ & Adapt & OptAdapt & FIBO & Sobol \\
\hline $10^{3}$ & $8.32 \mathrm{e}-03$ & $1.73 \mathrm{e}-03$ & $1.42 \mathrm{e}-03$ & $5.32 \mathrm{e}-02$ \\
\hline $10^{4}$ & $1.05 \mathrm{e}-03$ & $8.05 \mathrm{e}-05$ & $7.33 \mathrm{e}-04$ & $6.31 \mathrm{e}-03$ \\
\hline $10^{5}$ & $2.42 \mathrm{e}-03$ & $6.32 \mathrm{e}-06$ & $7.42 \mathrm{e}-04$ & $4.73 \mathrm{e}-03$ \\
\hline $10^{6}$ & $5.45 \mathrm{e}-05$ & $7.58 \mathrm{e}-07$ & $2.71 \mathrm{e}-04$ & $5.54 \mathrm{e}-04$ \\
\hline \hline
\end{tabular}

MC approach is better than the deterministic method when the dimensionality increases. The advantage of the optimized adaptive algorithm in comparison with the previously used adaptive algorithm is shown on Figure 1, and the computation of the position of the signs and the peak are given in Figure 2. The numerical results including relative errors and computational times corresponding to the algorithms under consideration are presented, and the algorithms efficiency is discussed. A numerical comparison for a given number of samples between the adaptive approach (Adapt) used in [13], the Sobol (Sob) and the Lattice sequences FIBO described in [13] and the new optimized Adaptive approach (OptAdapt) has been given in Tables II-VII. From the all experiments it can be clearly seen that the optimized adaptive approach gives relative errors with at least 1 or 2 orders better than those produced by the adaptive approach, because of the increased number of subregions taken in every subdomain $M$. The adaptive approach itself gives superior results to the other two stochastic approaches as it is completely described in our previous study [13]. The optimized Adaptive MC approach outperforms the other two approaches FIBO and Sobol QMC by at least 3 orders even for 18 dimensional case, see Table VII. We should emphasize here that the efficiency of the optimized adaptive $\mathrm{MC}$ algorithm under consideration is high when computational peculiarities of the integrand occur only in comparatively small subregion of the initial integration domain as it is in the case of the Wigner kernel.

\section{CONCLUSIONS}

The optimized adaptive Monte Carlo algorithm under consideration gives the most accurate results in computing the Wigner kernel by a stochastic approach and it has lower computational complexity than the existing deterministic approaches. This means that the proposed optimized stochastic approach is of great importance for the problems in quantum mechanics with high dimensions. Therefore, the presented optimized adaptive MC algorithm is one new successful solution (in terms of robustness and reliability) of Richard Feynman's problem for Wigner kernel evaluation for dimension $d>12$.

\section{REFERENCES}

[1] Berntsen J., Espelid T.O., Genz A. (1991) An adaptive algorithm for the approximate calculation of multiple integrals, ACM Trans. Math. Softw. 17: 437-451.

[2] Dimov I. (2008) Monte Carlo Methods for Applied Scientists, New Jersey, London, Singapore, World Scientific, 291 p., ISBN-10 981-022329-3.

[3] Dimov I., Karaivanova A., Georgieva R., Ivanovska S. (2003) Parallel Importance Separation and Adaptive Monte Carlo Algorithms for Multiple Integrals, Springer Lecture Notes in Computer Science, 2542, 99-107.

[4] Dimov I., Georgieva R. (2010) Monte Carlo Algorithms for Evaluating Sobol' Sensitivity Indices. Math. Comput. Simul. 81(3): 506-514.

[5] Ermakov S.M. (1985) Monte Carlo Methods and Mixed Problems, Nauka, Moscow.

[6] Feynman R.P. (1948) Space-time approach to non-relativistic quantum mechanics, Rev. Mod. Phys. 20.

[7] Sellier J.M. (2015) A signed particle formulation of non-relativistic quantum mechanics, Journal of Computational Physics 297: 254-265.

[8] Sellier J.M., Dimov I. (2016) On a full Monte Carlo approach to quantum mechanics, Physica A: Statistical Mechanics and its Applications Volume 463: 45-62.

[9] Sellier J.M., Dimov I. (2014) The many-body Wigner Monte Carlo method for time-dependent ab-initio quantum simulations, J. Comput. Phys. 273: 589-597.

[10] Sellier J.M., Nedjalkov M., Dimov I. (2015) An introduction to applied quantum mechanics in the Wigner Monte Carlo formalism, Physics Reports Volume 577: 1-34.

[11] Shao S., Lu T., Cai W. (2011) Adaptive conservative cell average spectral element methods for transient Wigner equation in quantum transport. Commun. Comput. Phys., 9: 711-739.

[12] Shao S. and Sellier J.M. (2015) Comparison of deterministic and stochastic methods for time-dependent Wigner simulations. J. Comput. Phys., 300: 167-185.

[13] Todorov, V., Dimov, I., Georgieva, R., \& Dimitrov, S. (2019). Adaptive Monte Carlo algorithm for Wigner kernel evaluation. Neural Computing and Applications, 1-12.

[14] Xiong Y., Chen Z., Shao S (2016) An advective-spectral-mixed method for time-dependent many-body Wigner simulations. SIAM J. Sci. Comput., to appear, [arXiv:1602.08853].

[15] Wigner E. (1932) On the quantum correction for thermodynamic equilibrium, Phys. Rev. 40: 749. 